\title{
RANDOM SCHRÖDINGER OPERATORS ON LONG BOXES, NOISE EXPLOSION AND THE GOE
}

\author{
BENEDEK VALKÓ AND BÁLINT VIRÁG
}

\begin{abstract}
It is conjectured that the eigenvalues of random Schrödinger operators at the localization transition in dimensions $d \geq 2$ behave like the eigenvalues of the Gaussian Orthogonal Ensemble (GOE). We show that there are sequences of $n \times m$ boxes with $1 \ll m \ll n$ so that the eigenvalues in low disorder converge to Sine $_{1}$, the limiting eigenvalue process of the GOE. For the GOE case, this is the first example where Wigner's famous prediction is proven rigorously: we exhibit a complex system whose eigenvalues behave like those of random matrices.
\end{abstract}

\section{INTRODUCTION}

When Wigner 20] introduced random matrices to model large atomic nuclei, his main goal was to find a simple mathematical model that shows repulsion between eigenvalues as observed in the data. The Gaussian Orthogonal Ensemble (GOE) has since been a remarkable success in the physics literature: several important complex systems are predicted to have eigenvalue repulsion akin to the GOE, most notably the Laplacians of many planar domains; see Bohigas et al., 4].

Given all the non-rigorous theories that predict GOE behavior, as well as much numerical evidence, it is surprising that there are hardly any mathematically rigorous results in this direction. Most objects with rigorously known bulk GOE behavior are themselves random matrices constructed similarly to the Gaussian Orthogonal Ensemble; see Tao and Vu [18] and Erdös et al. 6] for recent breakthrough results in this direction.

The goal of this paper is to provide the first example where Wigner's prediction is proven rigorously: a complex system whose eigenvalues behave like those of random matrices. To clarify, the Gaudin-Mehta theorem implies that the rescaled eigenvalue process of the $n \times n$ GOE matrix converges to a limiting point process, which we call Sine $_{\beta}, \beta=1$; the same holds for GUE and GSE matrices, with $\beta=2,4$, respectively (see Valkó and Virág [19] for general $\beta$ ). A model is called (bulk) universal if its eigenvalue process converges to $\operatorname{Sine}_{\beta}$, for $\beta=1,2$ or 4 .

We show that certain random Schrödinger operators on long $m \times n$ boxes, $1 \ll$ $m \ll n$, behave like the Gaussian Orthogonal Ensemble. More precisely, we will show

Theorem 1. There exists a sequence of $m \times n$ boxes, $1 \ll m \ll n$, so that the rescaled eigenvalue process of the adjacency matrix plus suitable diagonal noise converges to the Sine 1 point process.

Received by the editors December 13, 2011 and, in revised form, October 2, 2012.

2010 Mathematics Subject Classification. Primary 60B20, 81Q10.

(C) 2014 American Mathematical Society 
Theorem 11 will be proved in Corollary 11, which is based on Proposition 9 (see Section 5 .

The boxes we consider are very long, so in some ways our result is related to the quasi-one-dimensional setting, except that both dimensions of the box converge to infinity.

Recall that the Sine 1 point process is the limit of the eigenvalues at the bulk of the spectrum of the Gaussian Orthogonal Ensemble; see Mehta [14]. The processlevel convergence described here implies convergence of eigenvalue gap sizes and all similar local statistics.

Lack of true universality in one dimension. We would like to put the present work in the context of two results. The first is due to Killip and Stoiciu [12]. They consider the CMV matrices with random Verblunski coefficients, which are a unitary analogue of random Schrödinger operators. Their focus is the CMV representation of the Haar unitary random matrix. The entries of this matrix are built out of the Verblunski coefficients, which in this case are independent but non-identically distributed complex random variables with spherically symmetric distributions. Naturally, since these are conjugates of Haar unitary matrices, the eigenvalue process converges to the Sine $_{2}$ process, the bulk limit of the GUE. Moreover, Killip and Stoiciu [12] show that this holds even if the distribution of the random variables is changed, as long as spherical symmetry and the variances are kept. In this sense, this is a universality result. (A similar result for the Hermitian case was proven by Valkó and Virág [19, but there, due to the lack of spherical symmetry, more moments need to be fixed.)

However, in one dimension, a very special, non-i.i.d. variance structure was necessary to get the Sine 2 limit. In fact Killip and Stoiciu [12] show that a constant factor change for all variables will change the limiting process (they call the limit $C \beta E$, later shown to be identical to $\left.\operatorname{Sine}_{\beta}\right)$. Many limiting processes can be achieved by adjusting the variances judiciously. It is expected that the more natural model, in which the variances are identical, would give a version of the $\operatorname{Sch}_{\tau}$ process, described below.

The standard critical one-dimensional Schrödinger operators were treated in Kritchevski et al. 13: we considered adding i.i.d. diagonal noise with variance $\sigma^{2} / n$ to the adjacency matrix of a one-dimensional path of length $n$. In this critical regime, as $n \rightarrow \infty$ we got a limiting point process $\operatorname{Sch}_{\tau}$, whose parameter is given by $\tau=\sigma^{2} /\left(2-\lambda_{*}^{2}\right)$, where $\lambda_{*}$ is the macroscopic location of our window in the spectrum. The key is that $\mathrm{Sch}_{\tau}$ is not a limit of GOE, GUE, or other usual random matrix models. It is a different point process with stronger repulsion and long-range order. As $\tau$ ranges from 0 to $\infty$, it interpolates between the clock and Poisson distributions. In Kritchevski et al. 13 it is also shown that for a special sequence of decaying variances, all $\mathrm{Sine}_{\beta}$ processes can also be achieved as limits. Again, this is not true universality: GOE-like limits appear only for a careful choice of variances.

Universality when $1 \ll m \ll n$. As we have seen, in order to find truly universal GOE/GUE phenomenon in Schrödinger operators, one has to go beyond one dimension. This is what we do here, by considering an $m \times n$ box, with $1 \ll m \ll n$ with diagonal noise added. 
Our strategy is similar to that of Katz and Sarnak [11, who proved the first true universality result for GUE for zeta functions of random algebraic curves over finite fields. There are two parameters, the degree $n$ and characteristic $p$. They first let $p \rightarrow \infty$ and use the Deligne equidistribution theorem to show that the zeros of that zeta function have the same distribution as eigenvalues of a Haar unitary matrix. They then conclude universality from a double limit argument (Section 13.8).

We proceed similarly. We first find a regime where as $n \rightarrow \infty$ the eigenvalues of our operator converge to that of a certain $m \times m$ random matrix which is similar, but not identical, to the GOE. Then, using results of Erdős, Yau, et al. and a diagonalization argument, we show that for a certain sequence of $1 \ll m \ll n$, we get the universal Sine $_{1}$ limit.

Random Schrödinger operators. The concept of localization for random Schrödinger operators was introduced by Anderson [2]. As more and more diagonal noise is added to the Laplacian of a large box, the eigenvectors change from being spread out over the entire box to localized on smaller regions. It is believed (see, for example, Altshuler and Shklovski [1] and Efetov [5]) that near this transition the eigenvalue process is GOE-like, and, as the noise increases, it is approximately Poisson.

In the long box case, we have a related phenomenon. As $n \rightarrow \infty$, we get a limiting point process $\Lambda_{m, \sigma}$ depending on the noise. In a certain scaling, when $\sigma \rightarrow 0$, this $\Lambda_{m, \sigma}$ converges to the eigenvalue process of an $m \times m$ Gaussian random matrix. More precisely, we have

Theorem 2 (Eigenvalue process transition). Consider boxes in $\mathbb{Z}^{2}$ with fixed base $\mathbb{Z}_{m}$ and fix a weight $0<r \leq 1$. Consider the process of eigenvalues of

$$
n\left(\left(r \mathbb{Z}_{m}\right) \times \mathbb{Z}_{n}+\frac{\sigma}{\sqrt{n}} V-I \lambda^{*}\right),
$$

where $V$ are diagonal matrices with independent, mean zero, variance 1 entries with a bounded third moment. Then

- For almost all $\lambda^{*} \in(-2(1-r \cos (\pi /(m+1))), 2(1-r \cos (\pi /(m+1))))$, for appropriate subsequences, this process has a limit $\Lambda_{\sigma}$ which depends on $m, r$ and $\lambda^{*}$.

- As $\sigma \rightarrow 0$, the process $\sigma^{-1} \Lambda_{\sigma}$ converges to the (randomly shifted) eigenvalue process of a random matrix with independent real Gaussian entries.

- For every $\sigma$, the process $\Lambda_{\sigma}$ is the zero process of the determinant of an $m$ dimensional matrix-valued analytic function described by a stochastic differential equation.

Remark 3. To illustrate the transition to Poisson, it is possible to show that

- as $\sigma \rightarrow \infty$ the process $\Lambda_{\sigma}$ converges to the Poisson point process.

We plan to do this in a subsequent article.

The proofs of both theorems are based on the analysis of transfer matrices. The proof of Theorem 2 is given in Section 4 It is based on Proposition 4 and Corollary 6, which are proved in the same section.

The noise term in the transfer matrix evolution in this scaling regime for block Jacobi matrices has been studied in the paper by Römer and Schulz-Baldes [15] using a language different from SDEs. The first arxiv version of the present paper was followed by the preprint of the paper by Bachmann and De Roeck [3], who, in 
independent work, also study SDE limits of transfer matrices. Their starting point is the so-called DMPK theory in the physics literature (due to Dorokhov-MelloPereyra- Kumar), which is essentially the study of diffusive limits of quasi-onedimensional random Schrödinger operators from a slightly different point of view. We refer the reader to the paper by Bachmann and De Roeck 3 . for a discussion of this theory. One of the novelties of our approach is that it allows studying the dependence on the eigenvalue $\lambda$, which in turn allows us to deduce the scaling limit of the spectrum, the main focus here.

Noise explosion. The central reason for the appearance of random matrices is noise explosion. For a simple case of this, consider

$$
B_{\eta, n}(t)=\frac{1}{\sqrt{n}} \sum_{k=0}^{\lfloor n t\rfloor} e^{i \eta k} X_{k},
$$

where the $X_{k}$ are independent random variables with, say, standard normal distribution. If $\eta=0(\bmod 2 \pi)$, then the sequence of functions $B_{\eta, n}$ converges in law to real Brownian motion. Otherwise, $B_{\eta, n}$ converges to complex Brownian motion. Thus a one-dimensional noise process $X_{k}$ gives rise to a two real-dimensional Brownian motion in the presence of oscillatory terms. Moreover, if $\eta, \nu$ are linearly independent over the integers, then $B_{\eta, n}, B_{\nu, n}$ converge jointly to two independent Brownian motions.

Now we change the setting so that the $X_{k}$ are $m \times m$ diagonal matrices with independent standard normal entries. Let $U$ be a unitary matrix with eigenangles that are linearly independent over the integers, and also assume that the absolute squares of the eigenvectors of $U$ are not orthogonal to each other. Now consider the sum

$$
B_{n}(t)=\frac{1}{\sqrt{n}} \sum_{k=0}^{\lfloor n t\rfloor} U^{-k} X_{k} U^{k} .
$$

A simple computation shows that $B_{n}(t)$ converges to a Hermitian matrix-valued Brownian motion process which is $m^{2}$-real-dimensional. In such oscillatory sums, noise that was originally restricted to the diagonal explodes into all possible directions, and changes dimension from $m$ to $m^{2}$. This phenomenon, which we call noise explosion, plays a central role in the proofs below.

The method of analysis in this paper is an extension of the one-dimensional case introduced in a different setting by Valkó and Virág [19] and further refined and simplified by Kritchevski et al. [13. The latter paper studies the most natural one-dimensional random Schrödinger operator at the localization transition.

Open problems. The current paper does not give any bounds on the aspect ratio of the long boxes $m / n$. This would require rates of convergence estimates for diffusion approximation.

Problem 1. Show that the result in this paper holds for $n=O\left(m^{q}\right)$ for some $q \geq 1$. What is the smallest possible value of $q$ for which the results hold? Is it $q=1$ ?

Problem 2. Extend the results to long boxes of higher dimension. The eigenvalue structure of the base here is more complicated; see Remark 12. 
Our results apply for weighted boxes, and the larger the weight is, the stronger the restrictions are on the centering $\lambda_{*}$. In particular, when $r=1$ we will need to take a sequence of $\lambda_{*} \rightarrow 0$ to get the Sine 1 process in the limit (see Corollary 11). However, the results should hold on a dense subset of $[-2,2]$.

Problem 3. Show that for any fixed weight $r$ the results hold for almost every $\lambda_{*} \in(-2,2)$.

\section{Description of the model And notation}

Let $\mathbb{Z}_{n}$ denote the graph of a path of length $n$ with vertices $1,2, \ldots, n$. We will use the same variables for a (weighted) graph and its adjacency matrix, so $\mathbb{Z}_{n}$ will also denote the $n \times n$ matrix with entries $\left(\mathbb{Z}_{n}\right)_{i, j}=1_{\{|i-j|=1\}}$. Let $\times$ denote the product of weighted graphs (or matrices), namely

$$
(A \times B)_{\left(i, i^{\prime}\right),\left(j, j^{\prime}\right)}=\mathbf{1}_{i^{\prime}=j^{\prime}} A_{i, j}+\mathbf{1}_{i=j} B_{i^{\prime}, j^{\prime}} .
$$

With tensor product, it can be expressed as $A \otimes I+I \otimes B$. For a positive real $r$ and weighted graph (or matrix) $G$ we will denote by $r G$ the graph where the weights are all multiplied by $r$ (which is the same as multiplying the matrix $G$ by $r$ ).

Weighted boxes. The boxes we consider are $\left[r \mathbb{Z}_{m}\right] \times \mathbb{Z}_{n}$ for some positive real $r$, where $n$ is typically much larger than $m$. This is the adjacency matrix of a weighted graph on the box $\mathbb{Z}_{m} \times \mathbb{Z}_{n}$, where the edges in the first direction have weight $r$ and the edges in the second direction have weight 1.

The adjacency matrix corresponds to Dirichlet boundary conditions. For a probabilistic interpretation, the matrix

$$
r \mathbb{Z}_{m} \times \mathbb{Z}_{n}-2(r+1) I_{m \times n}
$$

is the transition probability matrix of the continuous time random walk on the box killed when it leaves; the jump rates are $r$ and 1 in the two different kinds of directions.

Some of our results will apply to $G \times \mathbb{Z}_{n}$, where $G$ is a general symmetric matrix with non-negative entries.

Chaoticity. For the noise explosion phenomenon to work, we need to consider conjugation by special angles. Consider points (angles) $\mathcal{X}=\left\{x_{1}, \ldots, x_{m}\right\}$ on the unit circle $\mathbb{R} / 2 \pi$. We will be interested in the set

$$
\begin{aligned}
\mathcal{A}:= & \left\{x_{i_{1}}+x_{i_{2}}+x_{i_{3}}+x_{i_{4}}\right\} \cup\left\{x_{i_{1}}+x_{i_{2}}-x_{j_{1}}-x_{j_{2}}: i_{k} \neq j_{\ell}\right\} \\
& \cup\left\{x_{i_{1}}+x_{i_{2}}+x_{i_{3}}-x_{j_{1}}: i_{k} \neq j_{1}\right\},
\end{aligned}
$$

where the indices run through $1, \ldots, m$ and the operations are meant $\bmod 2 \pi$. The chaoticity of $\mathcal{X}$ is defined as the distance of the set $\mathcal{A}$ from 0 :

$$
\operatorname{cha}(\mathcal{X})=\operatorname{dist}(0, \mathcal{A})
$$

We call the $\mathcal{X}$ chaotic if it has non-zero chaoticity.

In particular, if the $\mathcal{X}$ is chaotic, then the $x_{i}$ 's are distinct. Moreover, if the $x_{i}$ 's are linearly independent over the integers, then they are chaotic. A condition very similar to chaoticity appears as the main assumption in the paper by Schulz-Baldes [16].

The noise explosion described in the introduction will rely on the following quantities. Let $G=O D O^{-1}$ be the diagonalization of $G$, so that $O$ is orthogonal 


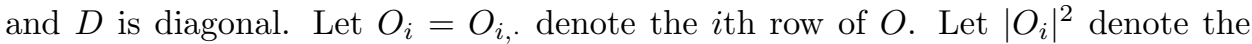
vector formed by the squares of the entries of $O_{i}$.

When $G$ is $\mathbb{Z}_{m}$ (i.e. its adjacency matrix), then the eigenvalues are given by $d_{j}=2 \cos (\pi j /(m+1))$. The eigenvectors are of the form

$$
O_{j k}=\sqrt{2 /(m+1)} \sin (\pi j k /(m+1)),
$$

and it is easy to check that

$$
(m+1)\left\langle\left|O_{i}\right|^{2},\left|O_{j}\right|^{2}\right\rangle= \begin{cases}3 / 2 & \text { for } i=j, \\ 1 & \text { for } i \neq j .\end{cases}
$$

\section{The REgularized transfer MATRIX EVOLUtion}

For this section, consider the operator $G \times \mathbb{Z}_{n}$ where $G$ is a symmetric matrix of dimension $m \times m$.

Suppose that we want to solve the equation $M u=\lambda u$ where $M=G \times \mathbb{Z}_{n}+V$ with a diagonal matrix $V$. We will do this by solving the system of linear equations recursively, slice by slice (where a slice is a copy of $G$, i.e. $G \times\{i\}$ ). Let $u$ be a function from the vertices of $G \times \mathbb{Z}_{n}$ to $\mathbb{R}$, and let $u_{i}(j)=u(j, i)$ so that $u_{i}$ is a vector indexed by $G$. Denote by $V_{i}$ the diagonal matrix which is the restriction of $V$ to the indices $G \times\{i\}$. Then the eigenvalue equation for entries with the same first coordinate $2 \leq i \leq n-1$ reads

$$
\lambda u_{i}=\left(G+V_{i}\right) u_{i}+u_{i-1}+u_{i+1},
$$

and if we set $u_{0} \equiv u_{n+1} \equiv 0$, then (44) holds for $i=1$ and $n$ as well. It is not hard to check that this system of $n$ equations is then equivalent to $M u=\lambda u$. Note that (44) can be written in terms of block transfer matrices of dimension $2 m$ as follows:

$$
\left[\begin{array}{c}
u_{k+1} \\
u_{k}
\end{array}\right]=\left[\begin{array}{cc}
\lambda I-G-V_{k} & -I \\
I & 0
\end{array}\right]\left[\begin{array}{c}
u_{k} \\
u_{k-1}
\end{array}\right] .
$$

Denoting the above matrix by $T_{k}$, we can now characterize the eigenvalues of $M$ as (5)

$$
\left\{\lambda: \exists u_{1}, u_{n} \text { such that } T_{n} \cdots T_{2} T_{1}\left[\begin{array}{c}
u_{1} \\
0
\end{array}\right] \|\left[\begin{array}{c}
0 \\
u_{n}
\end{array}\right]\right\}=\left\{\lambda: \operatorname{det}\left(\left(T_{n} \cdots T_{2} T_{1}\right)_{(1,1)}\right)=0\right\} .
$$

Here the subscript $(1,1)$ refers to the top left $m \times m$ submatrix, and both representations are equivalent to this submatrix having some eigenvector $u_{1}$ with eigenvalue 0 . So we may write

$$
\operatorname{spec}(M)=\operatorname{zeros}_{\lambda}\left[\operatorname{det}\left(\left(T_{n} \cdots T_{2} T_{1}\right)_{(1,1)}\right)\right],
$$

where $\operatorname{spec}(\cdot)$ will refer to the eigenvalue counting measure and $\operatorname{zeros}_{\lambda}$ refers to the zero counting measure of a real-analytic function of $\lambda$. It is not hard to check that the equality also holds in the presence of multiple zeros.

We now introduce the unperturbed version of the transfer matrix:

$$
T_{*}=\left[\begin{array}{cc}
\lambda_{*} I-G & -I \\
I & 0
\end{array}\right],
$$

where $\lambda_{*}$ is some fixed reference point. Let $G=O D O^{T}$ be the diagonalization of $G$ with $O$ orthogonal and $D=\operatorname{diag}\left(d_{1}, \ldots, d_{m}\right)$ diagonal. We will use the shortened notation $X^{Y}=Y^{-1} X Y$ which means $G=D^{O^{T}}$. 
We may change basis to study products of

$$
T_{k}^{O \otimes I_{2}}=\left[\begin{array}{cc}
\lambda I-D-V_{k}^{O} & -I \\
I & 0
\end{array}\right], \quad O \otimes I_{2}=\left[\begin{array}{cc}
O & 0 \\
0 & O
\end{array}\right],
$$

and note that

$$
T_{*}^{O \otimes I_{2}}=\left[\begin{array}{cc}
\lambda_{*} I-D & -I \\
I & 0
\end{array}\right] .
$$

This matrix can be completely diagonalized as it is just the permutation of a block diagonal matrix with $2 \times 2$ blocks. (We have learned that such a change of basis has been considered for a slightly different problem by Schulz-Baldes [16.) Let

$$
z_{j}=\left(\lambda_{*}-d_{j}\right) / 2+i \sqrt{1-\left(\left(\lambda_{*}-d_{j}\right) / 2\right)^{2}} \text { and } Z=\operatorname{diag}\left(z_{1}, \ldots, z_{m}\right) .
$$

We assume that $\left|\lambda_{*}-d_{j}\right| \in(0,2)$ for all $j$, which means that $z_{j}$ is a unit length complex number in the upper half plane.

Then we have

where

$$
T_{*}^{\left(O \otimes I_{2}\right) Q}=\left[\begin{array}{cc}
\bar{Z} & 0 \\
0 & Z
\end{array}\right]
$$

$$
S=\left[\begin{array}{cc}
\operatorname{diag}\left|\bar{z}_{j}-z_{j}\right|^{-1 / 2} & 0 \\
0 & \operatorname{diag}\left|\bar{z}_{j}-z_{j}\right|^{-1 / 2}
\end{array}\right], \quad Q=\left[\begin{array}{cc}
\bar{Z} & Z \\
I & I
\end{array}\right] S
$$

and

Then the matrix

$$
Q^{-1}=i S\left[\begin{array}{rr}
I & -Z \\
-I & \bar{Z}
\end{array}\right]
$$

$$
X_{k}=\left(T_{*}^{-k} T_{k} \cdots T_{2} T_{1}\right)^{\left(O \otimes I_{2}\right) Q}
$$

is deterministic matrix factors away from the transfer matrix evolution $T_{k} \cdots T_{1}$. However, it has the advantage that, unlike the product $T_{k} \cdots T_{1}$, it changes slowly as $k$ varies. This can be seen from its evolution. From the definition we have

$$
X_{k}=\left(T_{*}^{-k} T_{k} T_{*}^{-1} T_{*}^{k}\right)^{\left(O \otimes I_{2}\right) Q} X_{k-1} .
$$

Note that in our case $T_{k}$ will be a small perturbation of $T_{*}$.

We call $\left(X_{k}, 0 \leq k \leq n\right)$ the regularized transfer matrix evolution, regularized at $\lambda_{*}$. In the next section, we will show that this evolution has a stochastic differential equation limit. But first let us check how to read the eigenvalues of the operator $M$ from $X_{n}$. By (6) the eigenvalues of $M$ are given by

$$
\begin{aligned}
& \left\{\lambda: \operatorname{det}\left(\left(\left(\left(T_{*}^{\left(O \otimes I_{2}\right) Q}\right)^{n} X_{n}\right)^{Q^{-1}\left(O^{-1} \otimes I_{2}\right)}\right)_{(1,1)}\right)=0\right\} \\
& =\left\{\lambda: \operatorname{det}\left(\left(\left(\left(T_{*}^{\left(O \otimes I_{2}\right) Q}\right)^{n} X_{n}\right)^{Q^{-1}}\right)_{(1,1)}\right)=0\right\} .
\end{aligned}
$$

Note that $\left(T_{*}^{\left(O \otimes I_{2}\right) Q}\right)^{n} X_{n}$ is a matrix of the form

$$
\left[\begin{array}{ll}
\bar{Z}^{n} X_{11} & \bar{Z}^{n} X_{12} \\
Z^{n} X_{21} & Z^{n} X_{22}
\end{array}\right]
$$

and so the $(1,1)$ block of $Q\left(T_{*}^{\left(O \otimes I_{2}\right) Q}\right)^{n} X_{n} Q^{-1}$ is given by

$$
\begin{aligned}
& i S_{11}\left(\bar{Z}^{n+1} X_{11}-\bar{Z}^{n+1} X_{12}+Z^{n+1} X_{21}-Z^{n+1} X_{22}\right) S_{11} \\
& =i S_{11}\left(\bar{Z}^{n+1}, Z^{n+1}\right) X_{n}(I,-I)^{T} S_{11} .
\end{aligned}
$$


Since $S_{11}$ is non-singular, we have

$$
\begin{aligned}
\operatorname{spec}(M) & =\operatorname{zeros}_{\lambda}\left[\operatorname{det}\left(\left(\bar{Z}^{n+1}, Z^{n+1}\right) X_{n}(I,-I)^{T}\right)\right] \\
& =\operatorname{zeros}_{\lambda}\left[\operatorname{det} \Im\left(\bar{Z}^{n+1}\left(\left(X_{n}\right)_{11}-\left(X_{n}\right)_{12}\right)\right)\right] .
\end{aligned}
$$

The second equality follows from the fact that the determinant is zero only for real $\lambda$ (as these are eigenvalues of a symmetric matrix $M$ ), and that for real $\lambda$, we have $X_{22}=\bar{X}_{11}$ and $X_{21}=\bar{X}_{12}$. This will be shown in the proof of Proposition 4 in the next section.

\section{The Limiting transfer MATRIX EVOlution}

The goal of this section is to show that the regularized transfer matrix evolution introduced in the previous section has a stochastic differential equation limit. It will follow that the eigenvalues of the corresponding operator converge in distribution to an explicitly constructible limit.

We first state the scaling limit of the evolution of transfer matrices.

Proposition 4 (Limiting evolution of transfer matrices). Fix $r, \sigma>0$. Consider the operator $M_{n}=r G \times Z_{n}+n^{-1 / 2} \sigma V$, where $V=V^{(n)}$ is a diagonal matrix with independent random entries of mean 0, variance 1 and uniformly bounded third moments. Denote the eigenvalues of $G$ by $d_{1}, \ldots, d_{m}$ and assume that $0<$ $\left|\lambda_{*}-r d_{j}\right|<2$ for all $j$ and that the angles $\arccos \left(\left(\lambda_{*}-r d_{j}\right) / 2\right), j=1, \ldots, m$, are chaotic. Consider the regularized transfer matrix evolution (9) corresponding to $\lambda_{*}$ : $\left(X_{k}^{(n)}, 0 \leq k \leq n\right)$.

Then for any finite $\Lambda \subset \mathbb{C}$ we have convergence in distribution as $n \rightarrow \infty$ :

$$
\left(X_{\lfloor n t\rfloor}^{(n)}\left(\lambda^{*}+\lambda / n\right), t \in[0,1], \lambda \in \Lambda\right) \Rightarrow\left(Y_{t}(\lambda), t \in[0,1], \lambda \in \Lambda\right),
$$

where $Y=Y_{t}(\lambda)$ is the strong solution of the $S D E$ :

$$
d Y=S^{2}\left[\begin{array}{cc}
I & 0 \\
0 & -I
\end{array}\right] i \lambda d t Y+i \sigma S\left[\begin{array}{cc}
d A & d B \\
-d \bar{B} & -d \bar{A}
\end{array}\right] S Y, \quad Y_{0}=I,
$$

and $S$ is defined in (8). Moreover, for any fixed $t$, the functions $\lambda \rightarrow X_{\lfloor n t\rfloor}^{(n)}\left(\lambda^{*}+\lambda / n\right)$ converge in distribution to the random analytic function $Y_{t}(\cdot)$ with respect to the uniform-compact topology of functions. The covariance structure of the matrixvalued Brownian motions $A(t), B(t)$ is as follows. We have

$$
A_{i j}=\bar{A}_{j i}, \quad B_{i j}=B_{j i}
$$

( $B$ is complex symmetric, not Hermitian) and

$$
\mathbf{E}\left|B_{i j}\right|^{2}=\mathbf{E} A_{i i} A_{j j}=\mathbf{E} A_{i i} \bar{A}_{j j}=\mathbf{E}\left|A_{i j}\right|^{2}=\left\langle\left|O_{i}\right|^{2},\left|O_{j}\right|^{2}\right\rangle t
$$

(where $O_{i}$ are defined in Section 2), and all covariances that do not follow from the above are zero.

Remark 5. In the case $G=\mathbb{Z}_{m}$ by (3) we have

$$
A(1)=\frac{1}{\sqrt{m+1}}\left(A^{\prime}+\zeta I\right), \quad B(1)=\frac{1}{\sqrt{m+1}} B^{\prime} .
$$

Here $A^{\prime}$ is a version of the GUE with $A_{i, j}^{\prime}=\bar{A}_{j, i}^{\prime}$ standard complex normals for $i \neq j$ and mean zero variance $1 / 2$ i.i.d. real normals in the diagonal. (The usual GUE would have variance 1 in the diagonal.) The random variable $\zeta$ is standard normal and independent of $A^{\prime}$. 
$B^{\prime}$ is an independent symmetric matrix with i.i.d. standard complex normal entries above and below the diagonal, and i.i.d. mean zero complex normals with variance $3 / 2$ in the diagonal (which are independent of everybody else).

Next, we consider the eigenvalues of the operators $M_{n}$.

Corollary 6. Consider the operators $M_{n}$ of Proposition 4 . Assume that along some subsequence $\bar{Z}^{n+1} \rightarrow Z_{*}$ as $n \rightarrow \infty$, where $Z$ is defined in (7) and $Z_{*}$ is a fixed $m \times m$ matrix. Then on this subsequence we have

$$
\operatorname{spec}\left(n\left(M_{n}-\lambda^{*} I\right)\right) \Rightarrow \operatorname{zeros}_{\lambda}\left[\operatorname{det}\left(\left(\bar{Z}_{*}, Z_{*}\right) Y_{1}(\lambda)(I,-I)^{T}\right)\right] .
$$

We proceed with the proof of Proposition 4. The proof relies on the noise explosion phenomenon introduced in Section 1.

Proof of Proposition 4. From now on, with a slight abuse of notation, we will use $X_{k}^{(n)}(\lambda)$ for $X_{k}^{(n)}\left(\lambda^{*}+\lambda / n\right)$ and sometimes we will drop the dependence on $\lambda$ and/or $n$.

We first study the evolution without scaling time. From (10)

$$
X_{k}-X_{k-1}=\left(T_{*}^{\left(O \otimes I_{2}\right) Q}\right)^{-k}\left(T_{k} T_{*}^{-1}-I\right)^{\left(O \otimes I_{2}\right) Q}\left(T_{*}^{\left(O \otimes I_{2}\right) Q}\right)^{k} X_{k-1} .
$$

The coefficient of $X_{k-1}$ above is given by

$$
R_{k}=\left[\begin{array}{cc}
Z^{k} & 0 \\
0 & Z^{-k}
\end{array}\right] Q^{-1}\left[\begin{array}{cc}
0 & \frac{\lambda}{n} I+\frac{\sigma}{\sqrt{n}} V_{k}^{O} \\
0 & 0
\end{array}\right] Q\left[\begin{array}{cc}
Z^{-k} & 0 \\
0 & Z^{k}
\end{array}\right] .
$$

Note that the vector $\left(X_{k}(\lambda): \lambda \in \Lambda\right)$ is a Markov chain in $k$. We can represent it as a $4 m^{2}|\Lambda|$-dimensional complex vector where the entries are labeled with $(i, j, \lambda)$. In order to prove that it converges to the appropriate SDE we use Proposition 13 of Section 6.

We first rewrite $R_{k}=\frac{\sigma}{\sqrt{n}} R_{k}^{\prime}+\frac{\lambda}{n} R_{k}^{\prime \prime}$, where $R_{k}^{\prime}$ contains $V_{k}^{O}$ and $R_{k}^{\prime \prime}$ contains $I$ from the middle term of $R_{k}$. We first focus on the noise term $R_{k}^{\prime}$ which expands to

$$
R_{k}^{\prime}=i S\left[\begin{array}{cc}
Z^{k} & 0 \\
0 & Z^{-k}
\end{array}\right]\left[\begin{array}{cc}
I & -Z \\
-I & \bar{Z}
\end{array}\right]\left[\begin{array}{cc}
0 & V_{k}^{O} \\
0 & 0
\end{array}\right]\left[\begin{array}{cc}
\bar{Z} & Z \\
I & I
\end{array}\right]\left[\begin{array}{cc}
Z^{-k} & 0 \\
0 & Z^{k}
\end{array}\right] S,
$$

the three middle factors simplify and we get

$$
R_{k}^{\prime}=i S\left[\begin{array}{cc}
Z^{k} V_{k}^{O} Z^{-k} & Z^{k} V_{k}^{O} Z^{k} \\
-Z^{-k} V_{k}^{O} Z^{-k} & -Z^{-k} V_{k}^{O} Z^{k}
\end{array}\right] S
$$

(the diagonal block entries are negative conjugates of each other (note that $V_{k}^{O}$ is real) and so are the off-diagonals). For the convergence to the limit, we need to understand the covariance of the partial sums of $R_{k}$ over $k$. (This is needed for condition (30) in Proposition 13, )

For this, we may ignore the $S$ factors for the moment, and study

$$
A_{\ell}=\sum_{k=1}^{\ell} Z^{k} V_{k}^{O} Z^{-k}, \quad B_{\ell}=\sum_{k=1}^{\ell} Z^{k} V_{k}^{O} Z^{k} .
$$

The $i, j$ entry of the first term is

$$
\sum_{k=1}^{\ell}\left(z_{i} \bar{z}_{j}\right)^{k} \sum_{r} O_{j, r}^{T} v_{k, r} O_{r, i}=\sum_{r} \sum_{k=1}^{\ell} v_{k, r}\left(z_{i} \bar{z}_{j}\right)^{k} O_{r, j} O_{r, i} .
$$


Consider the complex covariance of the $i, j$ and $i^{\prime}, j^{\prime}$ entries in $A_{\ell}$. Since that matrix is Hermitian, we may assume $i \leq j$ and $i^{\prime} \leq j^{\prime}$. Since the $v$ 's are independent, the covariance is given by

$$
\begin{aligned}
\mathbf{E} A_{i, j} \bar{A}_{i^{\prime}, j^{\prime}} & =\sum_{k=1}^{\ell}\left(z_{i} \bar{z}_{j} \bar{z}_{i^{\prime}} z_{j^{\prime}}\right)^{k} \sum_{r} O_{r, j} O_{r, i} O_{r, j^{\prime}} O_{r, i^{\prime}} \\
& = \begin{cases}\ell\left\langle\left|O_{i}\right|^{2},\left|O_{j}\right|^{2}\right\rangle & \text { for }(i, j)=\left(i^{\prime}, j^{\prime}\right), \\
\ell\left\langle\left|O_{i}\right|^{2},\left|O_{i^{\prime}}\right|^{2}\right\rangle & \text { for }\left(i, i^{\prime}\right)=\left(j, j^{\prime}\right), \\
\mathcal{O}(1) & \text { otherwise. }\end{cases}
\end{aligned}
$$

This is because we can write $z_{i} \bar{z}_{j} \bar{z}_{i^{\prime}} z_{j^{\prime}}=e^{i \alpha}$ with $|\alpha| \leq \pi$, and unless $\alpha=0$, the sum will be $\mathcal{O}\left(|\alpha|^{-1}\right)$. By the chaoticity condition $\alpha=0$ can happen only if the conjugated $z_{i}$ are matched up in pairs to equal non-conjugated $z_{i}$, in which case the indices have to be the same. The only options are $i=i^{\prime}, j=j^{\prime}$ or $i=j, i^{\prime}=j^{\prime}$.

This explains that the correlation between an entry $A$ and the entry of $B$ is always $\mathcal{O}(1)$ : there will be an odd number of conjugated $z$ 's, so such a matching will not occur.

The expectation of the product on the other hand is given by

$$
\begin{aligned}
\mathbf{E} A_{i, j} A_{i^{\prime}, j^{\prime}} & =\sum_{k=1}^{\ell}\left(z_{i} \bar{z}_{j} z_{i^{\prime}} \bar{z}_{j^{\prime}}\right)^{k} \sum_{r} O_{r, j} O_{r, i} O_{r, j^{\prime}} O_{r, i^{\prime}} \\
& = \begin{cases}\ell\left\langle O_{i}^{2}, O_{i^{\prime}}^{2}\right\rangle & \text { for } i=j \text { and } i^{\prime}=j^{\prime}, \\
\mathcal{O}(1) & \text { otherwise } .\end{cases}
\end{aligned}
$$

Indeed, these are the only possible matchings for the conjugated and not-conjugated $z$ 's if $i \leq j$ and $i^{\prime} \leq j^{\prime}$. Now

$$
\mathbf{E} B_{i, j} B_{i^{\prime}, j^{\prime}}=\mathcal{O}(1),
$$

since no matching can occur in the relevant product $z_{i} z_{j} z_{i^{\prime}} z_{j^{\prime}}$. Finally, for $i \leq j$ and $i^{\prime} \leq j^{\prime}$ (since $B$ is a complex symmetric matrix),

$$
\mathbf{E} B_{i, j} \bar{B}_{i^{\prime}, j^{\prime}}=\left\{\begin{array}{ll}
\ell\left\langle O_{i}^{2}, O_{j}^{2}\right\rangle & \text { for } i=i^{\prime} \text { and } j=j^{\prime}, \\
\mathcal{O}(1) & \text { otherwise. }
\end{array} .\right.
$$

Turning to the drift term $R_{k}^{\prime \prime}$ we first simplify it to get

$$
R_{k}^{\prime \prime}=i S\left[\begin{array}{cc}
I & Z^{2 k} \\
-Z^{-2 k} & -I
\end{array}\right] S
$$

This leads to the estimate

$$
i \sum_{k=1}^{\ell} S\left[\begin{array}{cc}
I & Z^{2 k} \\
-Z^{-2 k} & -I
\end{array}\right] S=i S\left[\begin{array}{cc}
\ell I & \mathcal{O}(1) \\
\mathcal{O}(1) & -\ell I
\end{array}\right] S .
$$

Remark 7. From (16) and (18) it is clear that $R_{k}$ is of the form $\left[\begin{array}{cc}a & b \\ \bar{b} & \bar{a}\end{array}\right]$, and thus this will hold for $I+R_{k}$ as well. The product of such matrices will have the same structure, which explains the last assertion of Section 3 .

We now turn back to the proof of Proposition 4, For the proof of the convergence, we will use Proposition 13, for which we need to verify the conditions listed there. Note that the proposition deals with the convergence of $\mathbb{R}^{d}$-valued Markov chains, 
and here we are dealing with vectors with entries as $2 m \times 2 m$ complex matrices. As we have already mentioned, we can just treat our Markov chain as a $4 m^{2}|\Lambda|$ dimensional complex vector, and by taking real and imaginary parts we may apply the proposition. Note that Proposition 13 immediately extends to complex-valued Markov chains as well. The only modification is that one has to introduce

$$
\tilde{a}^{n}(t, x):=\mathbf{E}\left[Y_{\lfloor n t\rfloor}^{n}(x) \bar{Y}_{\lfloor n t\rfloor}^{n}(x)^{\mathrm{T}}\right]
$$

as well, and assume that $\tilde{a}$ satisfies the same conditions as $a$. This is because the covariance structure of a complex vector $Y$ can be computed from the quantities $\mathbf{E}(Y-\mathbf{E} Y)(Y-\mathbf{E} Y)^{T}$ and $\mathbf{E}(Y-\mathbf{E} Y)(\bar{Y}-\mathbf{E} \bar{Y})^{T}$.

In our case the conditional distribution of $X_{k+1}-X_{k}$, given $X_{k}=x$, is given by

$$
Y_{k}(x)=\left(T_{*}^{\left(O \otimes I_{2}\right) Q}\right)^{-k-1}\left(T_{k+1} T_{*}^{-1}-I\right)^{\left(O \otimes I_{2}\right) Q}\left(T_{*}^{\left(O \otimes I_{2}\right) Q}\right)^{k+1} x .
$$

The functions $a^{n}(t, x), \tilde{a}^{n}$ and $b^{n}(t, x)$ are defined according to (27) and (20):

$$
\begin{aligned}
& a^{n}(k / n, x)=\mathbf{E}\left[\left(Y_{k}(x)\right)_{i, j, \lambda}\left(Y_{k}(x)\right)_{i^{\prime}, j^{\prime}, \lambda^{\prime}} \mid X_{k}^{(n)}=x\right], \\
& \tilde{a}^{n}(k / n, x)=\mathbf{E}\left[\left(Y_{k}(x)\right)_{i, j, \lambda}\left(\bar{Y}_{k}(x)\right)_{i^{\prime}, j^{\prime}, \lambda^{\prime}} \mid X_{k}^{(n)}=x\right], \\
& b^{n}(k / n, x)=\mathbf{E}\left[\left(Y_{k}(x)\right)_{i, j, \lambda} \mid X_{k}^{(n)}=x\right] .
\end{aligned}
$$

The entries of $a, \tilde{a}$ are indexed by $(i, j, \lambda),\left(i^{\prime}, j^{\prime}, \lambda^{\prime}\right)$ and the entries of $b$ are indexed by $(i, j, \lambda)$.

- The boundedness of the cubic terms (29) is proved as follows. It is clear that $Y_{k}(x)$ is a bounded linear function of $\lambda$ 's and the $v_{i}$ 's with coefficients given by the entries of $x$. So as long as $x$ is bounded, condition (29) holds because of the third moment assumption on the $v_{i}$ 's. To ensure that $x$ is bounded, we first consider a truncated process in which $a^{n}, \tilde{a}^{n}$ and $b^{n}$ are multiplied by a smooth version of the cutoff function $\mathbf{1}(\|x\| \leq c)$. This will basically stop $X_{k}^{n}$ once $\left\|X_{k}^{n}\right\| \geq c$. It will follow from the proposition that the truncated process converges to the truncated version of the limit, for every $c>0$. However, as $c \rightarrow \infty$, the solution of the truncated SDE is with high probability equal to the non-truncated one. This follows from the fact that the SDE (12) is linear, and therefore it does not blow up in finite time. It follows that the truncated processes converge (12) from which we also get that the original processes must converge there as well.

- Conditions (28) and (30) follow from the calculations in the first part of the proof. We check the conditions for $a$ and $b$; for $\tilde{a}$ it will follow similarly.

Instead of working directly with $a$ and $b$, we introduce

$$
\hat{Y}_{k}=\frac{\lambda}{n}\left[\begin{array}{cc}
I & Z^{2 k} \\
-Z^{-2 k} & -I
\end{array}\right]+\frac{1}{\sqrt{n}}\left[\begin{array}{cc}
Z^{k} V_{k}^{O} Z^{-k} & Z^{k} V_{k}^{O} Z^{k} \\
-Z^{-k} V_{k}^{O} Z^{-k} & -Z^{-k} V_{k}^{O} Z^{k}
\end{array}\right]
$$

and let $\hat{a}^{n}(t)$ and $\hat{b}^{n}(t)$ be $n$ times the second and first moments of the $4 m^{2}|\Lambda|$-vector $\hat{Y}_{\lfloor n t\rfloor}$. Then we have

$$
X_{k}-x=i S \hat{Y}_{k} S x,
$$

given $X_{k-1}=x$. This is a linear function of $x$, and since $x$ is bounded by truncation, it suffices to check (30) for $\hat{a}$ and $\hat{b}$ rather than the original $a, b$. 
Similarly, (28) for (the truncated) $a, b$ is implied by

$$
\left|\hat{a}^{n}(t)\right|+\left|\hat{b}^{n}(t)\right| \leq c .
$$

This, in turn, follows from the expression for $\hat{Y}_{k}$ above (note that $Z$ is diagonal with unit complex numbers).

Returning to (30), we first check the existence of $\hat{b}$ so that

$$
\sup _{t}\left|\int_{0}^{t} \hat{b}^{n}(s) d s-\int_{0}^{t} \hat{b}(s) d s\right| \rightarrow 0 .
$$

This is clear by the following computation, based on (19):

$$
\hat{b}(k / n)=\sum_{\ell=1}^{k} \mathbf{E} \hat{Y}_{\ell}^{(n)}=\sum_{\ell=1}^{k} \frac{\lambda}{n}\left[\begin{array}{cc}
Z^{k} I Z^{-k} & Z^{k} I Z^{k} \\
-Z^{-k} I Z^{-k} & -Z^{-k} I Z^{k}
\end{array}\right]=\lambda \frac{k}{n}\left[\begin{array}{cc}
I & 0 \\
0 & -I
\end{array}\right]+\mathcal{O}\left(n^{-1}\right),
$$

where the $\mathcal{O}$ is uniform in $k$.

To check the second moment terms, we will consider the $\lambda=\lambda^{\prime}$ case; the general case is similar. We first note that since the mean increments are of $\mathcal{O}(1 / n)$, it suffices to look at the variance instead of the second moment. Thus we need the covariance matrix of (the vector version of)

$$
\frac{1}{\sqrt{n}}\left[\begin{array}{cc}
Z^{k} V_{k}^{O} Z^{-k} & Z^{k} V_{k}^{O} Z^{k} \\
-Z^{-k} V_{k}^{O} Z^{-k} & -Z^{-k} V_{k}^{O} Z^{k}
\end{array}\right] .
$$

This, by the computations above (starting at equation (16)), converges to the covariance matrix of the corresponding noise term in the SDE.

For the statement about the convergence of the analytic functions $X_{k}^{(n)}(\lambda)$, we will need a bound of the form

$$
\mathbf{E}\left\|X_{k}^{(n)}(\lambda)\right\| \leq f(\lambda, k / n)
$$

where $f(\lambda, t)$ is a locally bounded function. Then by Corollary 15 the claim follows.

We have

$$
\begin{aligned}
\mathbf{E}\left(\left\|X_{k}\right\|^{2}-\left\|X_{k-1}\right\|^{2} \mid \mathcal{F}_{k}\right) & =\mathbf{E} \operatorname{tr}\left(\Delta X X_{k-1}^{T}+\Delta X^{T} X_{k-1}+\Delta X \Delta X^{T}\right) \\
& \leq \frac{c\left(1+\lambda^{2}\right)}{n}\left\|X_{k-1}\right\|^{2} .
\end{aligned}
$$

Taking expectations we now see that

$$
\mathbf{E}\left\|X_{k}\right\|^{2}-\mathbf{E}\left\|X_{k-1}\right\|^{2} \leq \frac{c\left(1+\lambda^{2}\right)}{n} \mathbf{E}\left\|X_{k-1}\right\|^{2} .
$$

Rearranging and taking a product for $1, \ldots, k$ we immediately get the estimate

$$
\mathbf{E}\left\|X_{k}\right\|^{2} \leq\left(1+\frac{c\left(1+\lambda^{2}\right)}{n}\right)^{k}\left\|X_{0}\right\|^{2} \leq \exp \left(c\left(1+\lambda^{2}\right) \frac{k}{n}\right)\left\|X_{0}\right\|^{2},
$$

from which the bound (22) follows.

Proof of Corollary [6. By the Skorokhod embedding in Kallenberg [10, we can realize the distributional convergence of the matrix-valued analytic functions $X^{(n)}(\cdot) \Rightarrow$ $Y_{1}(\cdot)$ as almost sure convergence. In particular, along our subsequence, we have a.s.

$$
\operatorname{det}\left(\left(\bar{Z}^{n+1}, Z^{n+1}\right) X_{n}^{(n)}(I,-I)^{T}\right) \rightarrow \operatorname{det}\left(\left(\bar{Z}_{*}, Z_{*}\right) Y_{1}(\lambda)(I,-I)^{T}\right)
$$


uniformly on compacts, and the limit is analytic in $\lambda$. Note that for $\lambda=0$ the matrix in the determinant equals

$$
2 i \Im\left(\bar{Z}_{*}\left(Y_{1}(0)_{11}-Y_{1}(0)_{12}\right)\right),
$$

whose distribution is absolutely continuous with respect to the distribution of the GOE; this follows from the SDE (12). Thus the determinant as a function of $\lambda$ is not identically zero with probability 1 , and the zeros of the subsequence converge to the zeros of the limit almost surely in our realization. The distributional convergence follows.

In order to prove Theorem 2 we will also need the following lemma:

Lemma 8. Fix a $G$ with distinct eigenvalues $d_{1}, \ldots, d_{m}$ with $\max _{j}\left|d_{j}\right|=\tilde{d}$ and $f i x$ $0<r<2 / \tilde{d}$. Then for a.e. $\lambda_{*} \in(-(2-r \tilde{d}), 2-r \tilde{d})$ there is a sequence $n_{\nu}$ so that the points $q_{j}=\arccos \left(\left(\lambda_{*}-r d_{j}\right) / 2\right)$ are chaotic and we also have $\left(n_{\nu}+1\right) q_{j} \rightarrow 0$ $\bmod 2 \pi$ for all $1 \leq j \leq m$.

Proof. If $\lambda_{*} \in(-(2-r \tilde{d}), 2-r \tilde{d})$, then the vector $q=q\left(\lambda_{*}\right)=\left(\arccos \left(\left(\lambda_{*}-\right.\right.\right.$ $\left.\left.\left.r d_{j}\right) / 2\right)\right)_{j=1, \ldots, m}$ is well defined because of our conditions. We will show that for a.e. $\lambda^{*}$ in that interval the vector $q$ has no non-zero integer vector orthogonal to it. Then the set $\left\{q_{j}\right\}_{j=1, \ldots, m}$ is chaotic, and because the orbit $\{n q \bmod 2 \pi: n \geq 0\}$ is dense on the $m$-torus we can find a subsequence $n_{\nu}$ so that $n_{\nu} q_{j}$ converges to 0 $\bmod 2 \pi$ for all $j$.

It suffices to show that for any fixed non-zero integer vector $w$, only finitely many $\lambda$ has $q(\lambda) \cdot w=0$. Note that $\lambda \mapsto q(\lambda) \cdot w$ is an analytic function on the interval $(-(2-r \tilde{d}), 2-r \tilde{d})$, so it has finitely many zeros there or it has to be constant zero. We will show that the latter is not possible, which will finish the proof.

The function

$$
q_{j}^{\prime}(\lambda)=-\frac{1}{2 \sqrt{1-\frac{1}{4}\left(\lambda-r d_{j}\right)^{2}}}
$$

has singularities at $\lambda_{j}^{ \pm}= \pm 2-r d_{j}$. If $q(\lambda) \cdot w=0$ for all values of $\lambda$, then these singularities must cancel out. But this is impossible if $w \neq \underline{0}$, as the numbers $\lambda_{j}^{-}, \lambda_{j}^{+}, j=1, \ldots, m$, are all different: $-2-r d_{j}=2-r d_{k}$ would imply $4=$ $r\left(d_{j}-d_{k}\right) \leq 2 r \tilde{d}<4$.

Proof of Theorem 2. Fix $r$ and $m$ and consider $G=Z_{m}$. This has distinct eigenvalues $d_{j}$ with $\left|d_{j}\right| \leq 2 \cos (\pi /(m+1))$. Then we may apply Lemma 8 , and for almost every $\lambda^{*} \in(-2(1-r \cos (\pi /(m+1))), 2(1-r \cos (\pi /(m+1))))$ there is a sequence $n_{\nu}$ with the properties given by the lemma. Next we may apply Proposition 4 and Corollary [6] with $Z_{*}=I$ to show that the eigenvalues of (11) converge to

$$
\Lambda_{\sigma}:=\operatorname{zeros}_{\lambda}\left[\operatorname{det}\left((I, I) Y_{1}(\lambda)(I,-I)^{T}\right)\right],
$$

where $Y_{t}(\lambda)$ is the solution of the SDE (12). This proves the first and the third statements. For the convergence of $\sigma^{-1} \Lambda_{\sigma}$ we first note that with the notation $\hat{Y}_{t}(\lambda)=\sigma^{-1}\left(Y_{t}(\sigma \lambda)-I\right)$ we have

$$
\sigma^{-1} \operatorname{zeros}_{\lambda}\left[\operatorname{det}\left((I, I) Y_{1}(\lambda)(I,-I)^{T}\right)\right] \stackrel{d}{=} \operatorname{zeros}_{\lambda}\left[\operatorname{det}\left((I, I) \hat{Y}_{1}(\lambda)(I,-I)^{T}\right)\right] .
$$


From (12) it follows that $\hat{Y}$ satisfies

$$
d \hat{Y}=S^{2}\left[\begin{array}{cc}
I & 0 \\
0 & -I
\end{array}\right] i \lambda d t(\sigma \hat{Y}+I)+i S\left[\begin{array}{cc}
d A & d B \\
-d \bar{B} & -d \bar{A}
\end{array}\right] S(\sigma \hat{Y}+I), \quad \hat{Y}_{0}=0 .
$$

As $\sigma \rightarrow 0$ the solution of this SDE converges to the solution of the SDE with $\sigma=0$ (see e.g. Theorem 11.1.4 of Stroock and Varadhan [17]) which is just a matrix-valued Brownian motion with drift. In particular,

$$
\begin{aligned}
\operatorname{det}\left((I, I) \hat{Y}_{1}(\lambda)(I,-I)^{T}\right) & \rightarrow \operatorname{det}\left[(I, I) i S\left[\begin{array}{cc}
A(1)+\lambda & B(1) \\
-\bar{B}(1) & -\bar{A}(1)-\lambda
\end{array}\right] S(I,-I)^{T}\right] \\
& =\operatorname{det}\left[2 i S_{11}(\lambda I+\Re A(1)-\Re B(1)) S_{11}\right],
\end{aligned}
$$

the zeros of which are $\operatorname{spec}(\Re B(1)-\Re A(1))$. Here $A(1), B(1)$ are Gaussian matrices described in Remark 5. Note that $\Re B(1)-\Re A(1)$ can be written as $(m+1)^{-1 / 2}(K+$ $b I)$, where $b$ is a standard normal random variable, and $K$ is a real symmetric matrix with independent mean zero real normal entries so that

$$
\mathbf{E} K_{i j}^{2}= \begin{cases}1, & i \neq j, \\ 5 / 4, & i=j .\end{cases}
$$

The $b I$ term amounts to a random shift of the local eigenvalue process.

\section{GOE AS A LIMIT}

In order to get the GOE limit, we need to change the standard deviation $\sigma$ with $n$.

Proposition 9. Let $G$ be a weighted graph with distinct eigenvalues $d_{i}$, and fix $0<r<2 / \max _{j}\left|d_{j}\right|$. Suppose that

- the conclusion of Lemma 8 holds for $\lambda_{*}$, i.e. the critical angles

$$
q_{j}=\arccos \left(\left(\lambda_{*}-r d_{j}\right) / 2\right)
$$

exist, are chaotic and there is a sequence $n_{\nu}$ with $\left(n_{\nu}+1\right) q_{j} \rightarrow 0 \bmod 2 \pi$,

- $\sigma_{\nu}$ is a sequence with $\sigma_{\nu} \rightarrow 0$ and $\frac{1}{\sigma_{\nu}} \max _{j}\left|e^{i\left(n_{\nu}+1\right) q_{j}}-1\right| \rightarrow 0$,

- we have a sequence $V_{\nu}$ of diagonal perturbation matrices where the entries are independent, have mean 0 , variance 1 and uniformly bounded third moment.

Then the regularized transfer matrix evolution $X_{k}^{\nu}$ for the operator

$$
M_{\nu}=\frac{n_{\nu}}{\sigma_{\nu}}\left(r G \times \mathbb{Z}_{n_{\nu}}+\frac{\sigma_{\nu}}{\sqrt{n_{\nu}}} V_{\nu}-\lambda_{*} I\right)
$$

satisfies the following. For any finite $\Lambda \subset \mathbb{C}$ we have convergence in distribution for the regularized transfer matrices

$$
\left(\frac{1}{\sigma_{\nu}}\left(X_{\left\lfloor n_{\nu} t\right]}^{\nu}\left(\lambda^{*}+\lambda \sigma_{\nu} / n_{\nu}\right)-I\right), t \in[0,1], \lambda \in \Lambda\right) \Rightarrow\left(Y_{t}(\lambda), t \in[0,1], \lambda \in \Lambda\right),
$$

where

$$
Y=i S^{2}\left[\begin{array}{cc}
I & 0 \\
0 & -I
\end{array}\right] \lambda t+i S\left[\begin{array}{cc}
A & B \\
-\bar{B} & -\bar{A}
\end{array}\right] S
$$

Here $A, B$ are matrix-valued Brownian motions with covariance structure given by (13), (14). 
Moreover, the eigenvalue process of $M_{\nu}$ converges to the eigenvalues of

$$
\Re(B(1)-A(1)) .
$$

Remark 10. For the $G=\mathbb{Z}_{m}$ case the proposition shows that instead of letting $n \rightarrow \infty$ first and then $\sigma \rightarrow 0$ to get the eigenvalues of a Gaussian matrix as the limit of the spectrum (as in Theorem 2), we can achieve this by replacing $\sigma$ with $n$.

In the case when the graph is $\mathbb{Z}_{m}$, the matrix $\Re(B(1)-A(1))$ is the one we got in the proof of Theorem 2. It can be written as $(m+1)^{-1 / 2}(K+b I)$, where $b$ is a standard normal random variable, and $K$ is a version of the GOE: a real symmetric matrix with independent mean zero real normal entries with covariance structure (24). Note that for the GOE, the diagonal terms have variance 2. The distribution of the matrix $K$ is not invariant under orthogonal conjugation. The $b I$ term amounts to a random shift of the local eigenvalue process.

Proof. Let $\tilde{X}_{k}^{\nu}(\lambda)=\frac{1}{\sigma_{\nu}}\left(X_{\left\lfloor n_{\nu} t\right\rfloor}^{\nu}\left(\lambda^{*}+\lambda \sigma_{\nu} / n_{\nu}\right)-I\right)$. From (15) (and the following discussion) we get

$$
\tilde{X}_{k}-\tilde{X}_{k-1}=\sigma^{-1} R_{k}(\sigma \lambda)\left(\sigma \tilde{X}_{k}+I\right)=\left(\frac{1}{\sqrt{n}} R_{k}^{\prime}+\frac{\lambda}{n} R_{k}^{\prime \prime}\right)\left(\sigma \tilde{X}_{k}+I\right) .
$$

The proof of the SDE convergence of $\tilde{X}$ very closely follows the proof of Proposition 4. The only difference is that because of $\sigma \rightarrow 0$ the functions $a(t, x), \tilde{a}(t, x), b(t, x)$ will not depend on $x$; that is why we get the matrix-valued Brownian motion in the limit.

For the convergence of eigenvalues, we write

$$
\begin{aligned}
\frac{1}{\sigma_{\nu}}\left(\bar{Z}^{n+1}, Z^{n+1}\right)\left(X_{n_{\nu}}^{(n)}-I\right)(I & ,-I)^{T}=\frac{1}{\sigma_{\nu}}\left(\bar{Z}^{n+1}-I, Z^{n+1}-I\right) X_{n_{\nu}}^{(n)}(I,-I)^{T} \\
& +\frac{1}{\sigma_{\nu}}(I, I) \cdot(I,-I)^{T}+\frac{1}{\sigma_{\nu}}(I, I)\left(X_{n_{\nu}}^{(n)}-I\right)(I,-I)^{T} .
\end{aligned}
$$

Note that $I$ is the $m \times m$ identity matrix, except in $X_{n_{\nu}}^{(n)}-I$, where it is $2 m$ dimensional. The first term on the right converges to 0 since $X_{n_{\nu}}^{(n)}(I,-I)^{T}$ is tight and $\left(Z^{n+1}-I\right) / \sigma_{\nu} \rightarrow 0$ by assumption. The second term vanishes, so considering the third term we conclude that

$$
\frac{1}{\sigma_{\nu}}\left(\bar{Z}^{n+1}, Z^{n+1}\right)\left(X_{n_{\nu}}^{(n)}-I\right)(I,-I)^{T} \rightarrow(I, I) Y_{\lambda}(1)(I,-I)^{T}
$$

uniformly on compacts. For a real $\lambda$ this is equal to $2 S_{11}(\lambda I+\Re(A(1)-B(1))) S_{11}$ and its determinant will be zero exactly at the eigenvalues of $\Re(B(1)-A(1))$. From this, the second part of the proposition follows by the same arguments as in the proof of Corollary 6 .

Corollary 11. Fix $0<r<1$. Then for almost all $\lambda_{*} \in[-2(1-r), 2(1-r)]$ there exists a sequence of integers $m_{\nu} \rightarrow \infty, n_{\nu} \rightarrow \infty$, positive numbers $\tilde{\sigma}=\tilde{\sigma}_{\nu} \rightarrow 0$, noise matrices $V=V_{\nu}$ and coefficients $\gamma=\gamma_{\nu}$ so that the eigenvalue process of

$$
\gamma\left[\left(r \mathbb{Z}_{m}\right) \times \mathbb{Z}_{n}+\tilde{\sigma} V-\lambda_{*} I\right]
$$

as $\nu \rightarrow \infty$ converges locally to the Sine $e_{1}$ process. Here the noise matrices $V$ are diagonal, the entries are independent, have mean 0 , variance 1 and a uniformly bounded third moment.

Moreover, if $r=1$, then we may choose a sequence $\lambda_{*}=\lambda_{*}^{(\nu)}$ so that (26) converges to the Sine 1 process. 
Corollary 11 immediately proves Theorem 1 .

Proof. First suppose that $0<r<1$. Note that the eigenvalues $d_{j}$ of $\mathbb{Z}_{m}$ are distinct and $\max \left|d_{j}\right|=2 \cos (\pi /(m+1))<2$.

We consider a $\lambda_{*}$ so that the statement of Lemma 8 holds for all values of $m$. We start with a fixed $m$ and consider the appropriate sequence $n_{\nu}$. Since $\left(n_{\nu}+1\right) q_{j} \rightarrow 0 \bmod 2 \pi$ we can choose $\sigma_{\nu}=\sigma_{\nu, m} \rightarrow 0$ so that we also have $\frac{1}{\sigma_{\nu}} \max _{j}\left|e^{i\left(n_{\nu}+1\right) q_{j}}-1\right| \rightarrow 0$. Then we may apply Proposition 9 with $G=\mathbb{Z}_{m}$ which means that the eigenvalues of (25) $M_{\nu}$ converge to those of $(m+1)^{-1 / 2}(K+$ $b I)$ where $K, b$ are described in Remark 10 .

As $m \rightarrow \infty$, by the methods of Erdös et al. 7] the bulk eigenvalue process of the matrices $\sqrt{m}(K+b I)$ (see Remark [10) converges locally to the Sine 1 process. The proof of this statement will be expanded in Lemma 16 in the Appendix.

This means that with $\tilde{\sigma}_{\nu}=\sigma_{\nu} / \sqrt{n}$ and an appropriate $\gamma_{\nu}$ the eigenvalue process (here denoted $s_{m, n}$ ) of (26) converges to the spectrum $s_{m}$ of $\sqrt{m}(K+b I)$, and as $m \rightarrow \infty$ this will converge to the Sine ${ }_{1}$ process. In short, in the topology of weak convergence, we have

$$
\begin{aligned}
& s_{m, n} \rightarrow s_{m}, \quad n \rightarrow \infty, m \text { fixed, } \\
& s_{m} \rightarrow \text { Sine }_{1}, \quad m \rightarrow \infty \text {. }
\end{aligned}
$$

The standard diagonalization argument provides a sequence $n_{m} \rightarrow \infty$ so that $s_{m, n_{m}} \rightarrow$ Sine $_{1}$.

For the $r=1$ case note that for a fixed $m$ by Lemma 8 we can find a $\lambda_{*}^{(m)} \in$ $\left(-4 \sin ^{2}(\pi /(2+2 m)), 4 \sin ^{2}(\pi /(2+2 m))\right)$ for which Proposition 9 can be applied. Thus for each $m$ (with an appropriate $\lambda_{*}^{(m)}$ centering) the spectrum of the rescaled process (26) converges to that of $\sqrt{m}(K+b I)$ along an appropriate subsequence. The same diagonalization argument as in the $r<1$ case produces a sequence along which we have convergence to the Sine ${ }_{1}$ process.

Remark 12 (Higher-dimensional boxes). When $G=\mathbb{Z}_{m_{1}} \times \cdots \times \mathbb{Z}_{m_{d}}$, then the eigenvectors of $G$ are of the form

$$
O_{j_{1}, \ldots, j_{d}}\left(k_{1}, \ldots, k_{d}\right)=\prod_{\ell=1}^{d} \sqrt{\frac{2}{m_{\ell}+1}} \sin \left(\frac{\pi j_{\ell} k_{\ell}}{m_{\ell}+1}\right)
$$

and the corresponding eigenvalues are $d_{j_{1}, \ldots, j_{d}}=2 \sum_{\ell=1}^{d} \cos \left(\frac{j_{\ell} \pi}{1+m_{\ell}}\right)$. One can also calculate that

$$
\left(\prod_{\ell=1}^{d}\left(m_{\ell}+1\right)\right)\left\langle\left|O_{\underline{i}}\right|^{2},\left|O_{\underline{j}}\right|^{2}\right\rangle=\prod_{\ell=1}^{d}\left(1+\frac{1}{2} 1\left(i_{\ell}=j_{\ell}\right)\right) .
$$

If the eigenvalues are distinct, then Lemma 8 and Proposition 9 will still apply. However, the limiting symmetric real Gaussian matrix $\Re(B(1)-A(1))$ will have a more complicated covariance structure for $d>1$ than the one described in Remark 10. and the current results are not strong enough to imply that the bulk scaling limit will be the Sine 1 process.

One can check that in that case $\Re(B(1)-A(1))=M_{1}+M_{2}$, where $M_{1}$ is a constant times a GOE matrix and $M_{2}$ is an independent Gaussian matrix with a non-trivial covariance structure. Because of the component $M_{1}$ the local relaxation 
flow argument of Erdős et al. [7] will go through. The problem is caused by the fact that for the other component $M_{2}$ the strong local semicircle result is not available.

\section{ApPEndix}

6.1. SDE limit of Markov chains. The following is the main tool for proving convergence in the osciallatory setting. It is Proposition 23 in the paper by Valkó and Virág [19, and is based on Theorem 7.4.1 of Ethier and Kurtz [9].

Proposition 13. Fix $T>0$, and for each $n \geq 1$ consider a Markov chain

$$
\left(X_{\ell}^{n} \in \mathbb{R}^{d}, \ell=1, \ldots,\lfloor n T\rfloor\right) .
$$

Let $Y_{\ell}^{n}(x)$ be distributed as the increment $X_{\ell+1}^{n}-x$ given $X_{\ell}^{n}=x$. We define

$$
b^{n}(t, x)=n \mathbf{E}\left[Y_{\lfloor n t\rfloor}^{n}(x)\right], \quad a^{n}(t, x)=n \mathbf{E}\left[Y_{\lfloor n t\rfloor}^{n}(x) Y_{\lfloor n t\rfloor}^{n}(x)^{\mathrm{T}}\right] .
$$

Suppose that as $n \rightarrow \infty$ we have

$$
\begin{aligned}
\left|a^{n}(t, x)-a^{n}(t, y)\right|+\left|b^{n}(t, x)-b^{n}(t, y)\right| & \leq c|x-y|+o(1), \\
\sup _{x, \ell} \mathbf{E}\left[\left|Y_{\ell}^{n}(x)\right|^{3}\right] & \leq c n^{-3 / 2},
\end{aligned}
$$

and that there are functions $a, b$ from $\mathbb{R} \times[0, T]$ to $\mathbb{R}^{d^{2}}, \mathbb{R}^{d}$ respectively with bounded first and second derivatives so that

$$
\sup _{x, t}\left|\int_{0}^{t} a^{n}(s, x) d s-\int_{0}^{t} a(s, x) d s\right|+\sup _{x, t}\left|\int_{0}^{t} b^{n}(s, x) d s-\int_{0}^{t} b(s, x) d s\right| \rightarrow 0 .
$$

Assume also that the initial conditions converge weakly:

$$
X_{0}^{n} \Rightarrow X_{0} .
$$

Then $\left(X_{\lfloor n t\rfloor}^{n}, 0 \leq t \leq T\right)$ converges in law to the unique solution of the SDE,

$$
d X=b d t+g d B, \quad X(0)=X_{0},
$$

where $B$ is a d-dimensional standard Brownian motion and $g$ is any $C^{2}$ function satisfying $g g^{\mathrm{T}}=a$.

We need a result that strengthens the convergence of random analytic functions.

Proposition 14. Let $f_{n}$ be a sequence of random analytic functions on an open set $D \subset \mathbb{C}$ so that $\max _{A}\left|f_{n}\right|$ is tight for every closed ball $A \subset D$ and $f_{n} \Rightarrow f$ in the sense of finite-dimensional distributions. Then $f$ has a unique analytic version and $f_{n} \Rightarrow f$ in distribution with respect to local-uniform convergence.

Proof. Pick a countable dense set of points $D^{\prime} \subset D$ and let $\mathcal{A}$ be the countable set of closed balls $A \subset D$ with center in $D^{\prime}$ and rational radius. We can first find a subsequence so that the joint distribution of $\max _{A}\left|f_{n}\right|, A \in \mathcal{A}$, and $f_{n}(z), z \in D^{\prime}$, converges. By the Skorokhod embedding theorem of Kallenberg [10] we can realize the sequence $\left(f_{n}(z), z \in D^{\prime}\right)$ on a single probability space $\Omega$ so that almost surely $f_{n}(z) \rightarrow f(z)$ for all $z \in D^{\prime}$ and also $\max _{A}\left|f_{n}\right|$ converges (and so it is bounded) for all $A \in \mathcal{A}$. By continuity, we can define the $f_{n}$ as analytic functions on $D$ for the probability space $\Omega$. Then a.s. the following holds: for every compact set $B$ there exists a random constant $c_{B}$ with $\max _{z \in B, n}\left|f_{n}(z)\right| \leq C_{B}$. To see this, note that $B$ is covered by $\bigcup_{A \in \mathcal{A}}$ interior $(A)$, and since $B$ is compact we can choose a 
finite subcover. Then the sequence $f_{n}$ has at least one analytic limit in the sense of uniform-on-compacts convergence by Montel's theorem. This limit must agree with $f$ on all points $z \in D^{\prime}$, so it is unique.

By the above argument any sequence has a further subsequence that converges locally uniformly to some analytic $f$ in distribution. But the distribution of $f$ is determined by its finite-dimensional distributions, so the limit is unique.

Corollary 15. Let $f_{n}$ be random analytic functions on an open set $D \subset \mathbb{C}$ so that $\mathbf{E}\left|f_{n}(z)\right| \leq g(z)$ for a locally bounded function $g$. Assume that $f_{n}(z) \Rightarrow f(z)$ in the sense of finite-dimensional distributions. Then $f$ has a unique analytic version and $f_{n} \Rightarrow f$ in distribution with respect to local-uniform convergence.

Proof. Suppose that a closed disk $A$ is contained in a slightly bigger disk $B$ which is still contained in $D$. Then by Cauchy's integral theorem $\max _{A}\left|f_{n}\right| \leq C \int_{\partial B}\left|f_{n}(z)\right|$ and by the condition on $\mathbf{E}\left|f_{n}(z)\right|$ we get that $\mathbf{E} \max _{A}\left|f_{n}\right|$ is uniformly bounded. This implies that $\max _{A}\left|f_{n}\right|$ is tight for every closed ball $A \subset D$, and the claim follows from the proposition.

6.2. The point process limit of the modified GOE. Consider the $n \times n$ symmetric random matrix $H_{n}=n^{-1 / 2}(K+b I)$ where $b$ is a standard normal random variable, and $K$ is the version of the GOE considered in (24). We will show that the eigenvalue process of $H_{n}$ in the bulk converges locally to the Sine ${ }_{1}$ process, the bulk scaling limit of the GOE eigenvalue process. Bulk scaling means that we consider $\rho(\lambda) \sqrt{n}\left(H_{n}-\lambda I\right)$, where $|\lambda|<2$ and $\rho(\lambda)=\frac{1}{2 \pi} \sqrt{4-\lambda^{2}} 1_{|\lambda| \leq 2}$ is the semicircle density.

Lemma 16. For any $|\lambda|<2$ and compactly supported continuous test function $\Theta: \mathbb{R}^{k} \rightarrow \mathbb{R}$ we have

$$
\begin{aligned}
\int_{\mathbb{R}^{n}} d \alpha_{1} \ldots d \alpha_{n} \Theta\left(\alpha_{1}, \ldots, \alpha_{k}\right) \rho(\lambda)^{-k}( & \left.p_{H, n}^{(k)}-p_{G O E, n}^{(k)}\right) \\
& \times\left(\lambda+\frac{\alpha_{1}}{n \rho(\lambda)}, \ldots \lambda+\frac{\alpha_{k}}{\rho(\lambda) n}\right) \rightarrow 0 .
\end{aligned}
$$

Here $p_{H, n}^{(k)}$ is the $k$-point intensity function of the eigenvalues of $H_{n}$. The function $p_{G O E, n}^{(k)}$ is the same for the $n \times n$ GOE matrix with variances $2 / n$ and $1 / n$ on the diagonal and elsewhere, respectively.

Proof. Since the proof works exactly the same way for any $|\lambda|<2$, we will assume $\lambda=0$.

Our proof relies on the local relaxation flow arguments of Erdős et al. [7] and the strong local semicircle law proved in [8. The argument in a nutshell is the following: if we have a real symmetric random matrix whose eigenvalues are wellapproximated by the semicircle law locally, then by adding a small constant times an independent GOE matrix (i.e. by running Dyson's Brownian motion for a small time with our matrix as the initial condition) the scaled eigenvalue process of the resulting matrix will be close to the Sine $_{1}$ process.

We will use Theorem 2.3 of Erdös et al. 8. (see also the comments after the theorem) which provides a powerful quantitative version of the previous argument. Since we can write $\frac{1}{\sqrt{n}} K=K_{1}+K_{2}$ where $K_{1}$ is $1 / 2$ times a GOE and $K_{2}$ is an 
independent symmetric random matrix satisfying the conditions of the theorem, then for any $q>0$ we have

$$
\begin{aligned}
& \mid \int_{-\frac{q}{\sqrt{n}}}^{\frac{q}{\sqrt{n}}} \frac{d \mu \sqrt{n}}{2 q} \int_{\mathbb{R}^{k}} d \alpha_{1} \ldots d \alpha_{n} \Theta\left(\alpha_{1}, \ldots, \alpha_{k}\right) \rho(0)^{-k} \\
& \quad \times\left(p_{K, n}^{(k)}-p_{G O E, n}^{(k)}\right)\left(\mu+\frac{\alpha_{1}}{n \rho(0)}, \ldots, \mu+\frac{\alpha_{k}}{n \rho(0)}\right) \mid \leq C n^{-1 / 4+\varepsilon}\left(q^{-1}+q^{-1 / 2}\right) .
\end{aligned}
$$

(We apply the theorem with $E=0, b=q / \sqrt{n}, t=1 / 2, \varepsilon^{\prime} \ll 1$ and $\delta=1-\varepsilon^{\prime}$.) Since the spectrum of $H_{n}$ can be obtained by a random shift of the spectrum of $n^{-1 / 2} K$, we have

$$
p_{H, n}^{(k)}\left(\frac{\alpha_{1}}{n \rho(0)}, \ldots, \frac{\alpha_{k}}{n \rho(0)}\right)=\int_{-\infty}^{\infty} \frac{d b}{\sqrt{2 \pi n^{-1}}} e^{-\frac{b^{2} n}{2}} p_{K, n}^{(k)}\left(b+\frac{\alpha_{1}}{n \rho(0)}, \ldots, b+\frac{\alpha_{k}}{n \rho(0)}\right) .
$$

By Fubini for any non-negative function $F$ we have

$$
\int_{-\infty}^{\infty} \frac{d b}{\sqrt{2 \pi n^{-1}}} e^{-\frac{b^{2} n}{2}} F(b)=\int_{0}^{\infty} e^{-\frac{q^{2}}{2}} \sqrt{\frac{2}{\pi}} q^{2} \int_{-\frac{q}{\sqrt{n}}}^{\frac{q}{\sqrt{n}}} \frac{d b \sqrt{n}}{2 q} F(b) d q .
$$

Using this with (32) and (33) we get the upper bound

$$
\mid \text { left hand side of (31) } \mid \leq \int_{0}^{\infty} e^{-\frac{q^{2}}{2}} \sqrt{\frac{2}{\pi}} q^{2} C n^{-1 / 4+\varepsilon}\left(q^{-1}+q^{-1 / 2}\right) \leq C^{\prime} n^{-1 / 4+\varepsilon},
$$

which shows that the limit of the eigenvalue process of $H_{n}$ is the same as the limit of $G O E$ eigenvalues multiplied by $n$ and shifted by an independent Gaussian $b_{n}$ of variance $n$. We first condition on the sequence of these independent Gaussians, so the GOE eigenvalues are now centered at $-b_{n}$, which, on the semicircle scale, converges to 0 . In the classical literature, convergence is usually proved around a fixed window at $c,|c|<2$ (on the semicircle scale). The moving window case is rigorously proved by Valkó and Virág [19]. The lemma follows after removing the conditioning.

\section{ACKNOWLEDGEMENTS}

The authors thank Eugene Kritchevski, Alex Bloemendal, and László Erdős for useful discussions, and Hermann Schulz-Baldes for references. We also thank the anonymous referees for valuable comments and suggestions. The second author thanks the hospitality of the Banff International Research Station, where part of this work was conceived. This research was supported by the NSF Grant DMS-0905820 (the first author) and the NSERC discovery accelerator grant program and the Canada Research Chair program (the second author).

\section{REFERENCES}

[1] B. Altshuler and B. I. Shklovski (1986). Repulsion of energy levels and conductivity of metal samples. Sov. Phys. JETP, 64: 127-135.

[2] P. W. Anderson (1958). Absence of diffusion in certain random lattices. Phys. Rev., 109: 1492-1505.

[3] S. Bachmann and W. De Roeck, From the Anderson model on a strip to the DMPK equation and random matrix theory, J. Stat. Phys. 139 (2010), no. 4, 541-564, DOI 10.1007/s10955010-9947-2. MR2638927(2011g:82132) 
[4] O. Bohigas, M.-J. Giannoni, and C. Schmit, Characterization of chaotic quantum spectra and universality of level fluctuation laws, Phys. Rev. Lett. 52 (1984), no. 1, 1-4, DOI 10.1103/PhysRevLett.52.1. MR730191 (85f:58034)

[5] Konstantin Efetov, Supersymmetry in disorder and chaos, Cambridge University Press, Cambridge, 1997. MR:1628498(99m:82001)

[6] László Erdős, Benjamin Schlein, and Horng-Tzer Yau, Universality of random matrices and local relaxation flow, Invent. Math. 185 (2011), no. 1, 75-119, DOI 10.1007/s00222-010-03027. MR2810797 (2012f:60020)

[7] László Erdős, Benjamin Schlein, Horng-Tzer Yau, and Jun Yin, The local relaxation flow approach to universality of the local statistics for random matrices (English, with English and French summaries), Ann. Inst. Henri Poincaré Probab. Stat. 48 (2012), no. 1, 1-46, DOI 10.1214/10-AIHP388. MR2919197

[8] László Erdős, Horng-Tzer Yau, and Jun Yin, Rigidity of eigenvalues of generalized Wigner matrices, Adv. Math. 229 (2012), no. 3, 1435-1515, DOI 10.1016/j.aim.2011.12.010. MR2871147

[9] Stewart N. Ethier and Thomas G. Kurtz, Markov processes, Wiley Series in Probability and Mathematical Statistics: Probability and Mathematical Statistics, John Wiley \& Sons Inc., New York, 1986. Characterization and convergence. MR838085 (88a:60130)

[10] Olav Kallenberg, Foundations of modern probability, 2nd ed., Probability and its Applications (New York), Springer-Verlag, New York, 2002. MR1876169 (2002m:60002)

[11] Nicholas M. Katz and Peter Sarnak, Random matrices, Frobenius eigenvalues, and monodromy, American Mathematical Society Colloquium Publications, vol. 45, American Mathematical Society, Providence, RI, 1999. MR1659828 (2000b:11070)

[12] Rowan Killip and Mihai Stoiciu, Eigenvalue statistics for CMV matrices: from Poisson to clock via random matrix ensembles, Duke Math. J. 146 (2009), no. 3, 361-399, DOI 10.1215/00127094-2009-001. MR2484278(2009k:81087)

[13] Eugene Kritchevski, Benedek Valkó, and Bálint Virág, The scaling limit of the critical onedimensional random Schrödinger operator, Comm. Math. Phys. 314 (2012), no. 3, 775-806, DOI 10.1007/s00220-012-1537-5. MR2964774

[14] Madan Lal Mehta, Random matrices, 3rd ed., Pure and Applied Mathematics (Amsterdam), vol. 142, Elsevier/Academic Press, Amsterdam, 2004. MR2129906 (2006b:82001)

[15] Rudolf A. Römer and Hermann Schulz-Baldes, The random phase property and the Lyapunov spectrum for disordered multi-channel systems, J. Stat. Phys. 140 (2010), no. 1, 122-153, DOI 10.1007/s10955-010-9986-8. MR2651442 (2011g:82058)

[16] H. Schulz-Baldes, Perturbation theory for Lyapunov exponents of an Anderson model on a strip, Geom. Funct. Anal. 14 (2004), no. 5, 1089-1117, DOI 10.1007/s00039-004-0484-5. MR2105954 (2005g:47070)

[17] Daniel W. Stroock and S. R. Srinivasa Varadhan, Multidimensional diffusion processes, Grundlehren der Mathematischen Wissenschaften [Fundamental Principles of Mathematical Sciences], vol. 233, Springer-Verlag, Berlin, 1979. MR.532498 (81f:60108)

[18] Terence Tao and Van Vu, Random covariance matrices: universality of local statistics of eigenvalues, Ann. Probab. 40 (2012), no. 3, 1285-1315, DOI 10.1214/11-AOP648. MR2962092

[19] Benedek Valkó and Bálint Virág, Continuum limits of random matrices and the Brownian carousel, Invent. Math. 177 (2009), no. 3, 463-508, DOI 10.1007/s00222-009-0180-z. MR2534097(2011d:60023)

[20] E. P. Wigner (1957). Gatlinberg Conference on Neutron Physics, Oak Ridge National Laboratory Report, ORNL 2309: 59.

Department of Mathematics, University of Wisconsin-Madison, Madison, Wisconsin 53706

E-mail address: valko@math.wisc.edu

Department of Mathematics, University of Toronto, Ontario, Canada M5S 2E4

E-mail address: balint@math.toronto.edu 